\title{
Susceptibilidade larval de populações de Aedes aegyptie Culex quinquefasciatus a inseticidas químicos
}

\section{Larval susceptibility of Aedes aegypti and Culex quinquefasciatus populations to chemical insecticides}

\author{
Jairo Campos e Carlos F S Andrade \\ Departamento de Zoologia do Instituto de Biologia da Universidade Estadual de Campinas. Campi- \\ nas, $S P$, Brasil
}

\section{Descritores}

Aedes. Larva. Inseticidas. Resistência a inseticidas. Inseticidas

organofosforados. Controle de vetores. Controle de mosquitos.

\section{Keywords}

Aedes. Larva. Insecticides. Insecticide resistance. Insecticides organophosphate. Vector control. Mosquito control.

\section{Resumo}

\section{Objetivo}

Avaliar a susceptibilidade a inseticidas químicos de larvas de Culex quinquefasciatus e Aedes aegypti, provenientes de áreas sujeitas ou não a tratamentos de controle. Métodos

Foram coletadas larvas de mosquitos em uma área não sujeita a tratamentos com inseticidas (Campinas, SP) e em áreas sujeitas a esses tratamentos (Campo Grande, MS e Cuiabá, MT). Foram usados bioensaios com concentrações diagnóstico e concentrações múltipla de inseticidas organofosforados e piretróides, segundo padrão da Organização Mundial de Saúde, para avaliar a susceptibilidade dessas larvas.

\section{Resultados}

Ensaios com larvas de Culex quinquefasciatus de Campinas, SP, permitiram a suspeita de resistência à cipermetrina e evidenciaram resistência à ciflutrina. Larvas dessa espécie coletadas em Campo Grande, MS, e Campinas, SP, apresentaram resistência ao temephos. Para a colônia campineira desta espécie, foram estabelecidas as razões de resistência: $R_{50}=6,36$ e $R_{95}=4,94$, com base em linhagem susceptível padrão. Adicionalmente, os testes com Aedes aegypti mostraram susceptibilidade similar ao temephos em uma população de campo (Cuiabá, MT) e uma de laboratório.

Conclusões

Os resultados indicam resistência a organofosforado e piretróides em Culex quinquefasciatus, evidenciando a necessidade de avaliações e monitoramento da efetividade dos inseticidas a serem usados nos programas de controle de mosquitos.

\section{Abstract}

\section{Objective}

To evaluate the susceptibility to chemical insecticides of Culex quinquefasciatus and Aedes aegypt larvae from areas subjected to control treatments or not.

\section{Methods}

Bioassays for diagnostic concentration and multiple concentration were performed for organophosphate and pyrethroid insecticides according to World Health Organization parameters. The susceptibility was assessed for mosquito larvae collected from an area not subjected to chemical control (Campinas, State of São Paulo, SP) and from other areas (Campo Grande, Mato Grosso do Sul, MS, and

Trabalho baseado em tese de doutorado, apresentado ao Instituto de Biologia da Unicamp, em 2002. Recebido em 22/8/2002. Reapresentado em 21/3/2003. Aprovado em 7/4/2003. 
Cuiabá, Mato Grosso, MT), in Brazil, subjected to such treatments.

Results

Tests for Culex quinquefasciatus larvae from Campinas, SP, allowed suspicion of resistance to cypermethrin and gave evidence of resistance to cyfluthrin. Larvae of this species collected in Campo Grande, MS, and Campinas, SP, presented resistance to temephos. For the colony from the latter locality, the following resistance rates were established: $R R_{50}=6.36$ and $R R_{95}=4.94$, in relation to a standard susceptible strain. Moreover, tests for Aedes aegypti showed similar susceptibility to temephos for a field population from Cuiabá, MT, and a laboratory population.

\section{Conclusions}

The results indicate resistance of Culex quinquefasciatus to organophosphate and pyrethroid insecticides and make evident the need for evaluation and monitoring of the efficiency of insecticides to be used in mosquito control programs.

\section{INTRODUÇÃO}

As espécies sinantrópicas de mosquitos Aedes aegypti (Linn.) e Culex quinquefasciatus Say são de grande importância em Saúde Pública por estarem relacionadas, respectivamente, à transmissão da dengue e da filariose bancroftiana nas Américas. As recentes epidemias de dengue ocorridas no Brasil $(1998,2002)$ e a endemicidade da filariose constituem desafios administrativos e públicos no controle de mosquitos. No Brasil, já existe indicação de resistência em populações de $C x$ quinquefasciatus. ${ }^{2,9,12}$ Por exemplo, em Fortaleza, capital do estado do Ceará, foi registrada resistência a organofosforados para Cx. quinquefasciatus. ${ }^{12}$ Essa mesma espécie mostrouse ainda resistente no Rio de Janeiro ${ }^{9}$ e em São Paulo. ${ }^{2,3}$ Segundo indicação da Organização Mundial da Saúde, a concentração diagnóstico de temephos para larvas de $C x$. quinquefasciatus é de $0,002 \mathrm{ppm} \cdot{ }^{13} \mathrm{O}$ presente trabalho avalia a susceptibilidade de populações de campo e de laboratório de Ae. aegypti e $C x$. quinquefasciatus a um organofosforado e dois piretróides usados no controle de mosquitos.

\section{MÉTODOS}

As larvas de Ae. aegypti avaliadas provieram da colônia com quatro anos e meio, linhagem Aea-Unicamp (Departamento de Zoologia) e a F1 de material proveniente do Campus Universitário em Campinas, em maio de 2002, linhagem Aea-FEF. Para essa espécie, em Cuiabá, MT, foram avaliadas larvas parentais da linhagem Aea-Cuiabá, provenientes de ovos coletados em armadilhas pelo Centro de Controle de Zoonoses em 2000, Cuiabá, MT. Para as larvas de $C x$. quinquefasciatus avaliadas, o material foi de coletas realizadas no Campus da Universidade Federal do Mato Grosso do Sul (UFMS), em Campo Grande, MS, em maio de 1998, linhagem UFMS-R, e no Córrego Fundo, em Cuiabá, MT, em setembro de 2000, linhagem Cuiabá-R. Para Cx. quinquefasciatus, os locais de coleta em Campo Grande e Cuiabá apresentaram histórico de controle com organofosforados. Para as avaliações de $C x$. quinquefasciatus em Campinas, SP, utilizou-se material de coletas feitas no Campus Universitário em julho de 1999, linhagem UNI-R1, e em fevereiro de 2001, linhagem UNI-R2. Até aquela época, não haviam sido feitas aplicações de quaisquer produtos de controle no Campus. Como padrão de referência, foram usadas as linhagens Rockefeller de $A$ e. aegypti $i^{5}$ e a linhagem IAL-S (Instituto Adolfo Lutz, São Paulo) de Cx. quinquefasciatus, originária de Iguape, SP, em 1983. ${ }^{3}$

Os inseticidas usados foram o organofosforado temephos (Abate 500E, Larvin $1 \mathrm{G}$ e Fersol 1G) e os piretróides cipermetrina (Ciper 250CE) e ciflutrina (Solfac 5CE). A partir de uma dissolução estoque à concentração de 100 ppm i.a. desses produtos em água, foram feitas diluições adicionais em água destilada para se chegar às concentrações de trabalho. Para o organofosforado temephos foram realizados testes de concentração diagnóstico $(\mathrm{CD}=0,012 ; 0,04$ e 0,06 ppm i.a.) e teste de concentração múltipla $(\mathrm{CM}=0,00056$ a 0,016 ppm i.a.). Para os piretróides, foram realizados testes de concentração diagnóstico para a cipermetrina $(\mathrm{CD}=0,0096)$ e de concentração múltipla para a ciflutrina $(\mathrm{CM}=0,00075$ a 0,03$)$ (Tabela 1). Para o temephos, foram estabelecidas as concentrações letais mediana $\left(\mathrm{CL}_{50}\right)$ e as $\mathrm{CL}_{95}$ com larvas de Ae. aegypti (Aea-Uni e Aea-FEF) e Cx. quinquefasciatus (UNI-R2).

Os bioensaios foram feitos com larvas de Ae. aegypti e Cx. Quinquefasciatus, seguindo-se padrões previamente propostos. ${ }^{4,5,13}$ Usaram-se grupos de 20 larvas ou mais de terceiro e/ou quarto estádio em copos descartáveis, com as soluções dos inseticidas para três ou mais repetições. A mortalidade final foi avaliada 24 horas após o contato com os inseticidas. Os valores selecionados dos dados foram submetidos à análise probit, usando-se o programa POLO-PC.${ }^{10}$ Foi verificado, então, o paralelismo nas respostas e as 
razões de resistência ( $R R)$ foram calculadas para as $\mathrm{CL}_{50}$ e $\mathrm{CL}_{95}$, comparando-se os valores das linhagens padrão.

\section{RESULTADOS}

A Tabela 1 apresenta uma relação do material biológico utilizado e os resultados dos testes de susceptibilidade. Em ensaios com larvas parentais (linhagem UNI-R1) de Cx. quinquefasciatus coletadas em Campinas, SP, foi verificada baixa mortalidade (11\%) a uma formulação comercial de cipermetrina (CD de 0,0096 ppm i.a.). Da mesma forma, houve resistência em larvas F2 (linhagem UNI-R2) para a concentração diagnóstico ( $\mathrm{CD}=0,03$ ppm i.a.) do piretróide ciflutrina. Larvas de Cx. quinquefasciatus apresentaram sobrevivência de 6,3 e 11,9\% ao temephos $(\mathrm{CD}=0,04$ ppm i.a.) na linhagem UFMS-R e de $5 \%$ $(C D=0,012$ ppm i.a.) na linhagem Cuiabá-R. Para larvas da colônia (UNI-R2) desta espécie em Campinas foi estabelecida uma $\mathrm{CL}_{50}=0,0076$ ppm i.a. e uma
$\mathrm{CL}_{95}=0,0144$ ppm i.a. de temephos. Com base nos resultados da linhagem-padrão IAL-S, as razões de resistência foram de $\mathrm{RR}_{50}=6,36$ e $\mathrm{RR}_{95}=4,94$.

Avaliações para o temephos com a linhagem AeaCuiabá mostraram tolerância. Os testes realizados recentemente com larvas das linhagens $\mathrm{Aea}$-Unicamp (colônia de 4,5 anos) e Aea-FEF (F1, de campo) de Ae. aegypti indicaram também tolerância ao temephos. Quando comparadas com a linhagem Rockefeller, as razões de resistência destas linhagens foram $\mathrm{RR}_{50}=1,67$ e $\mathrm{RR}_{95}=1,85$ para Aea-Unicamp, $\mathrm{RR}_{50}=1,92$ $\mathrm{e} \mathrm{RR}_{95}=1,83$ para Aea-FEF; os coeficientes angulares (s) das linhas concentração-resposta foram 4,8 $\pm 0,6$ para $A e a$-Unicamp, 5,9 $\pm 0,7$ para $A e a-F E F$ e 5,5 $\pm 0,6$ para Rockefeller (Tabela 2).

\section{DISCUSSÃO}

$\mathrm{O}$ resultado do teste com cipermetrina em $C x$. quinquefasciatus (UNI-R1) levantou suspeita de re-

Tabela 1 - Linhagens de Culex quinquefasciatus, época dos ensaios e resultados das avaliações da susceptibilidade a inseticidas pelo uso de teste de concentrações diagnóstico ou teste de concentrações múltiplas.

\begin{tabular}{|c|c|c|c|c|c|}
\hline Linhagem (Data) & Estádio & $\begin{array}{l}\text { Tipo de } \\
\text { Teste } \\
\end{array}$ & Inseticida* & $\begin{array}{l}\text { Concentrações } \\
\text { ppm i.a. }\end{array}$ & $\begin{array}{l}\text { Mortalidade (DP)** } \\
\text { ou CL }_{\%} \text { (ppb i.a.) }\end{array}$ \\
\hline UFMS-R (maio/98) & $\begin{array}{l}3^{\circ} \\
4^{\circ}\end{array}$ & $2 \times C D$ & TEM 500E & $\begin{array}{c}0,04 \\
0,045\end{array}$ & $\begin{array}{c}88,09(14,87) \% \\
93,71(5,55) \%\end{array}$ \\
\hline UNI-R1（julho/99） & $4^{\circ}$ & $C D$ & CIP 250CE & 0,0096 & $11,17(6,26) \%$ \\
\hline Cuiabá-R (set/00) & $4^{\circ}$ & $C D$ & TEM 1G & $\begin{array}{c}0,012 \\
0,024 \\
0,06\end{array}$ & $\begin{array}{c}95 \% \\
100 \% \\
100 \%\end{array}$ \\
\hline UNI-R2 (mar/01) & $4^{\circ}$ & $\mathrm{CM}$ & CYF 5CE & 0,002 a 0,016 & $0,42(0,8) \%$ a $27,4(12,3) \%$ \\
\hline UNI-R2 (mar/01) & $4^{\circ}$ & CM & CYF 5CE & 0,012 a 0,03 & $12,3(5,9) \%$ a $47,5(9,6) \%$ \\
\hline UNI-R2 (jun/02) & $\begin{array}{l}3^{\circ} \\
4^{\circ}\end{array}$ & $\mathrm{CM}$ & TEM $1 \mathrm{G}$ & 0,004 a 0,016 & $\begin{array}{c}\mathrm{CL}_{50}=7,6(6,7-9)^{* * *} \\
\mathrm{CL}_{95}=14,4(11,3-24,8)\end{array}$ \\
\hline IAL-S (jun/02) & $\begin{array}{l}3^{\circ} \\
4^{\circ}\end{array}$ & CM & TEM $1 \mathrm{G}$ & 0,00056 a 0,004 & $\begin{array}{l}\mathrm{CL}_{50}=1,2(0,9-1,5) \\
\mathrm{CL}_{95}=2,9(2,1-5,6)\end{array}$ \\
\hline
\end{tabular}

CD: Concentração diagnóstico.

$\mathrm{CM}$ : Concentrações Múltiplas .

*TEM = temephos, $\mathrm{CIP}=$ cipermetrina, $\mathrm{CYF}=$ ciflutrina.

**(DP) desvio padrão.

***(Intervalo de Confiança $95 \%$ da $\left.\mathrm{CL}_{\%}\right)$.

Tabela 2 - Linhagens de Aedes aegypti, época dos ensaios e resultados das avaliações da susceptibilidade a inseticidas pelo uso de concentrações diagnóstico ou testes de concentrações múltiplas.

\begin{tabular}{|c|c|c|c|c|c|}
\hline Linhagem (Data) & Estádio & $\begin{array}{l}\text { Tipo de } \\
\text { Teste }\end{array}$ & Inseticida* & $\begin{array}{l}\text { Concentrações } \\
\text { ppm i.a. }\end{array}$ & 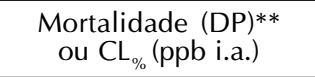 \\
\hline Cuiabá (set/00) & $3^{\circ}$ & $C D$ & TEM $1 \mathrm{G}$ & 0,012 & $100 \%$ \\
\hline Rockefeller (set/00) & $3^{\circ}$ & $C D$ & TEM $1 \mathrm{G}$ & $\begin{array}{l}0,012 \\
0,024\end{array}$ & $\begin{array}{l}100 \% \\
100 \%\end{array}$ \\
\hline Rockefeller (mar/01) & $3^{\circ}$ & $\mathrm{CM}$ & CYF 5CE & 0,0005 a 0,004 & $4,3(0,9) \%$ a $96,1(3,9) \%$ \\
\hline Rockefeller (mar/01) & $\begin{array}{l}4^{\circ} \\
3^{\circ} \\
4^{\circ}\end{array}$ & $\mathrm{CM}$ & CYF 5CE & 0,00075 a 0,006 & $12,8(7,9) \%$ a $100 \%$ \\
\hline Unicamp (jun/02) & $\begin{array}{l}3^{\circ} \\
4^{\circ}\end{array}$ & $\mathrm{CM}$ & TEM $1 \mathrm{G}$ & 0,005 a 0,02 & \multirow{3}{*}{$\begin{array}{c}\mathrm{CL}_{50}=9,8(8,6-11) * * * \\
\mathrm{CL}_{95}=21,7(18,1-28,8) \\
\mathrm{CL}_{50}=11,3(8,8-14,9) \\
\mathrm{CL}_{95}=21,4(15,8-48,3) \\
\mathrm{CL}_{50}=5,9(4,9-6,9) \\
\mathrm{CL}_{95}=11,7(9,3-18)\end{array}$} \\
\hline FEF (jun/02) & $\begin{array}{l}3^{\circ} \\
4^{\circ}\end{array}$ & $\mathrm{CM}$ & TEM $1 \mathrm{G}$ & 0,005 a 0,02 & \\
\hline Rockefeller (jun/02) & $\begin{array}{l}3^{\circ} \\
4^{\circ}\end{array}$ & $\mathrm{CM}$ & TEM $1 \mathrm{G}$ & 0,0025 a 0,01 & \\
\hline
\end{tabular}

*TEM= temephos, $\mathrm{CIP}=$ cipermetrina. $\mathrm{CYF}=$ ciflutrina.

**(DP) desvio padrão.

***(Intervalo de Confiança $95 \%$ da $\left.\mathrm{CL}_{\%}\right)$. 
sistência para esta linhagem. Considerou-se para isso que registros de letalidade com cipermetrina para essa espécie são maiores em concentrações menores: $\mathrm{CL}_{50}=0,0016$ ppm i.a. para larvas de uma linhagem susceptível e $\mathrm{CL}_{50}=0,004$ ppm i.a. para uma linhagem parental com resistência, em populações cubanas, ${ }^{1}$ e registro de uma $\mathrm{CL}_{50}=0,003$ e uma $\mathrm{CL}_{90}=0,01 \pm$ 0,006 ppm i.a. deste piretróide para uma colônia resistente de Cx. quinquefasciatus, no Rio de Janeiro, quando comparada com a $\mathrm{CL}_{50}=0,0008$ da linhagem de referência. ${ }^{9}$

Para o piretróide ciflutrina, poucos registros de testes são conhecidos com mosquitos. Uma RR de valor 4 pela $\mathrm{CL}_{50}$ em larvas de Ae. aegypti da Venezuela foi registrada quando comparada com a linhagem padrão Rockefeller ${ }^{8}$ indicando aumento das esterases como mecanismo envolvido na resistência aos piretróides na linhagem avaliada. Provas de susceptibilidade para este inseticida, em $C x$. quinquefasciatus da Venezuela, não registraram resistência. ${ }^{11}$ Nossas provas preliminares em $C x$. quinquefaciatus (UNIR2) indicaram resistência ao produto quando comparadas com a linhagem Rockefeller de Ae. Aegypti. Em geral, a sensibilidade aos inseticidas em linhagens padrão é maior para $C x$. quinquefasciatus do que para Ae. aegypti (Cutkomp \& Subramanyam, ${ }^{7}$ 1986; WHO, ${ }^{13}$ 1992), e se comparado com as $\mathrm{CL}_{50}$ da ciflutrina e da cipermetrina em Ae. aegypti (Campos $\&$ Andrade, ${ }^{5}$ 2001) e de cipermetrina e outros piretróides em $C x$. quinquefasciatus. ${ }^{1,9}$ Da mesma forma, os resultados das avaliações com a linhagem UNI$\mathrm{R} 2$ de $C x$. quinquefasciatus indicaram resistência baixa $\left(\mathrm{RR}_{99}<5\right)$ ao temephos quando comparada com a linhagem susceptível IAL-S $\left(\mathrm{CL}_{95}=0,0029\right.$ ppm i.a. $)$. A dose diagnóstico de temephos usada para $C x$. quinquefasciatus em Campo Grande foi 20 vezes superior àquela recomendada pela $\mathrm{WHO},{ }^{13} \mathrm{e} 13,8$ vezes superior à $\mathrm{CL}_{95}$ da IAL-S. Portanto, a sobrevivência indica real resistência da população ao inseticida. Já para as larvas de Cx. quinquefasciatus, em Cuiabá, foi encontrada resistência baixa a esse inseticida. Quando comparados os resultados de Cuiabá com os obtidos para a linhagem padrão de $C x$. quinquefasciatus (IAL) em Campinas, a priori teríamos uma $\mathrm{RR}_{95} \cong 4$.

Embora no presente trabalho não tenha sido identificado o mecanismo metabólico envolvido na resistência aos organofosforados, já foi registrada elevação das esterases em populações brasileiras de $C x$. quinquefasciatus. ${ }^{3,12}$ Têm sido observados marcadores cromossômicos de amplificação dos genes de esterases, associados à resistência aos organofosforados nas linhagens UNI-R1 e UNI-R2 (Campos et al ${ }^{6}$ ). Assim, as estratégias de controle de Cx. quinquefasciatus com organofosforados e piretróides em Campinas, Campo Grande e Cuiabá devem monitorar previamente sua eficiência. $\mathrm{O}$ desenvolvimento de resistência nessas populações pode estar associado ao uso indiscriminado de inseticidas nos ambientes domiciliar e agrícola, além dos tratamentos específicos em Campo Grande e Cuiabá.

Os resultados das avaliações com o temephos em larvas de Ae. aegypti de Cuiabá, MT, não mostraram resistência quando comparadas com a linhagem padrão Rockefeller. O monitoramento das populações de laboratório (4 anos após) e campo de Ae. Aegypti, em Campinas, não mostraram a resistência registrada em 1998 à $\mathrm{CD}=0,04$ ppm i.a. (Campos \& Andrade, ${ }^{5}$ 2001). Em função das pequenas diferenças, entre as duas populações, nas respostas ao temephos e da sobreposição dos intervalos de confiança, pode-se considerar que não há diferença entre as concentrações letais das linhagens. Logo, a susceptibilidade ao temephos $1 \mathrm{G}$ em Ae. aegypti não estaria comprometida, pelo menos para uso, se necessário, em pontos não estratégicos, como os avaliados em Campo Grande, Campinas e Cuiabá. Avaliações dos produtos comerciais são viáveis e devem ser uma prática no monitoramento.

Os resultados obtidos para as áreas sujeitas (Campo Grande e Cuiabá) ou não (Campinas) a tratamentos com inseticidas evidenciaram resistência baixa a estes produtos em $C x$. quinquefasciatus e susceptibilidade em Ae. aegypti. Desta forma, quando populações de campo são avaliadas e comparadas a populações padrão, as mudanças na resposta aos inseticidas $\left(\mathrm{CL}_{50}\right.$ e $\left.\mathrm{CL}_{95}\right)$ da ordem de até três vezes nas razões de resistência podem ser consideradas como tolerância; de 3 a 5 vezes, resistência baixa; de 5 a 10, moderada; de 10 a 20, média e acima de 20 , uma resistência alta Tal conhecimento pode ajudar no planejamento adequado do manejo da resistência e nas estratégias de controle a serem aplicadas em cada ponto, sem comprometer a eficiência dos produtos.

\section{AGRADECIMENTOS}

À Universidade Federal de Mato Grosso do Sul, à Universidade Estadual de Campinas e ao Centro de Controle de Zoonoses (Cuiabá, MT), pela permissão de uso de suas instalações e laboratórios. Ao médico veterinário Gerson Blatt e aos biólogos Leny Bezerra da Costa e Benedito Oscar Fernandes de Campos do Centro de Controle de Zoonoses (Cuiabá, MT), pela colaboração nas avaliações dos testes. Ao professor Délsio Natal da Faculdade de Saúde Pública da Universidade de São Paulo, por colocar a disposição a linhagem IAL. 


\section{REFERÊNCIAS}

1. Bisset J, Rodríguez M, Soca A. Cross-resistance to malathion in Cuba Culex. quinquefasciatus induced by larval selection with deltamethrin. Med Vet Entomol 1998;12:109-12.

2. Bracco JE, Dalbon M, Marinotti O, Barata JMS. Resistência a inseticidas organofosforados e carbamatos em população de Culex quinquefasciatus. Rev Saúde Pública 1997;31:182-3.

3. Bracco JE, Barata, JMS, Marinotti O. Evaluation of insecticide resistance in a population of Culex quinquefasciatus (Diptera: Culicidae) from São Paulo, Brazil. Mem Inst Oswaldo Cruz 1999;94:115-20.

4. Brown AWA. Insecticide resistance in mosquitoes: a pragmatic review. J Am Mosq Control Assoc 1986;2:123-40.

5. Campos J, Andrade CFS. Susceptibilidade larval de duas populações de Aedes aegypti a inseticidas químicos. Rev Saúde Pública 2001;35:232-6.

6. Campos J, Andrade CFS, Recco Pimentel SM. Malpighian tubule polytene chromosomes of Culex quinquefasciatus (Diptera, Culicinae). Mem Inst Oswaldo Cruz 2003;98:383-386.

7. Cutkomp LK, Subramanyam B. Toxicity of pyrethroids to Aedes aegypti larvae in relation to temperature. J Am Mosq Control Assoc 1986;3:347-9.
8. Fernández DM, Rodríguez M, Bisset J, Perez E. Identification of esterase resistance mechanisms in Aedes aegypti from Carabobo State, Venezuela in Summaries of Mosquito Vector Control and Biology in Latin America - a Ninth Symposium, St. Louis, MO, February 1999. J Am Mosq Control Assoc 1999;15:421.

9. González T, Bisset JA, Díaz C, Rodríguez MM, Brandolini MB. Insecticide resistance in a Culex quinquefasciatus strain from Rio de Janeiro, Brazil. Mem Inst Oswaldo Cruz 1999;94:121-2.

10. LeOra Software. Polo-PC, probit or logit analysis. Berkeley (CA); 1987.

11 Reyes-Lugo M, Neus $M$. Insecticide resistance in Culex quinquefascitus Say 1823 (Diptera: Culicidae) from Zulia State, Venezuela. Rev Cient-Fac Cienc Veter 2000;10:441-7.

12. Yébakima A, Yp-Tcha MM, Reiter P, Bisset J, Delay B, Chevillon C, Pasteur N. Detoxifying esterases in Culex pipiens quinquefasciatus from the caribbean countries. J Am Mosq Control Assoc 1995;11:363-6.

13. World Health Organization. Vector resistance to pesticides. Geneva; 1992. (WHO - Technical Report) 